\title{
Linx
}

Revue des linguistes de l'université Paris X Nanterre

$56 \mid 2007$

Linguistique des genres

\section{Épistémologie et genres du discours dans le cercle de Bakhtine}

Sheila Vieira de Camargo Grillo

\section{(2) OpenEdition}

Journals

Édition électronique

URL : http://journals.openedition.org/linx/355

DOI : $10.4000 /$ linx.355

ISSN : 2118-9692

Éditeur

Presses universitaires de Paris Nanterre

\section{Édition imprimée}

Date de publication : 1 juin 2007

Pagination : 19-36

ISSN : 0246-8743

\section{Référence électronique}

Sheila Vieira de Camargo Grillo, "Épistémologie et genres du discours dans le cercle de Bakhtine », Linx [En ligne], 56 | 2007, mis en ligne le 18 février 2011, consulté le 30 avril 2019. URL : http:// journals.openedition.org/linx/355; DOI : 10.4000/linx.355

Département de Sciences du langage, Université Paris Ouest 


\title{
Épistémologie et genres du discours dans le cercle de Bakhtine
}

\author{
Sheila Vieira de Camargo Grillo \\ Université de São Paulo - Capes - CNPq
}

Les travaux contemporains sur les genres du discours sont presque tous tributaires de l'approche du cercle de Bakhtine, témoignant de l'importance de son cadre théorique et de la puissance de ses catégories heuristiques. La lecture de la traduction du texte "Les genres du discours $»^{1}$ a influencé les développements contemporains des diverses approches du texte et du discours dans les pays francophones et au Brésil. Cependant, la place de la notion de genre dans l'ensemble du projet bakhtinien n'est, aujourd'hui encore, pas tout à fait claire. Les choix terminologiques de la traduction, le décalage temporel entre les différentes traductions, l'influence des théories françaises sur la réception du texte, la méconnaissance du contexte socio-historique de la production des textes bakhtiniens, constituent des difficultés qui entravent la compréhension du lecteur de la théorie bakhtinienne.

\footnotetext{
${ }^{1}$ On découvre les origines « apocryphes » de quelques textes de ce livre chez Shepperd (2005, p. 13) : «Le volontarisme textologique assez brutal de Kozinov ne peut que nous faire regretter de manière plus intense encore la décision des éditeurs du cinquième tome (dont Gogotisvili) d'exciser de la version publiée de "Problema recevyx zanrov» («Le Problème des genres du discours») de nombreuses citations (obligatoires à l'époque, donc quasiment privées de toute signification) du travail de Staline sur le marxisme en linguistique. Comme le dit Mika Lähteenmäki, 'une fois de plus, les recherches scientifiques sur Bakhtine doivent accepter le fait que notre compréhension de Bakhtine dépend toujours et est façonnée par les intérêts de ceux qui ont accès à ses archives et par les détenteurs des droits d'auteur de ces textes'».
} 
L'objectif de cet article est de repérer, en parcourant les textes disponibles de Bakhtine et de son cercle, le statut de la notion de genre du discours dans le projet épistémologique de fonder une étude dialogique du langage qui s'appellerait «métalinguistique ». Cette discipline ne vise pas à remplacer la linguistique ${ }^{2}$, mais envisage un programme de recherche sur des aspects du langage qui ne pourraient pas être traités par une approche hors contexte et basée sur un modèle scientifique galiléen $^{3}$.

La poursuite de cet objectif se fera au moyen des trois hypothèses de travail majeures : (1) la notion de genre présuppose la compréhension du dialogisme qui est l'objet d'étude du programme métalinguistique; (2) tous les aspects constitutifs du genre chez Bakhtine dépassent l'objet de la linguistique conçu à partir du Cours de linguistique générale de Saussure ; (3) la notion de genre est présente dans tout l'œuvre de Bakhtine et son Cercle, même si la traduction française a effacé sa présence. Cette dernière hypothèse implique que la compréhension des aspects constitutifs du concept de "genre » passe par la lecture des textes dont l'autorité, selon quelques chercheurs, n'est pas encore résolue ou, d'après d'autres, est déjà tranchée pour Voloshinov et Medvedev - autrement dit, que la diversité des auteurs n'empêche pas l'identification d'un projet commun de recherche dont le genre serait partie intégrante.

\section{La méta(ou trans)linguistique}

On retrouve parmi les textes bakhtiniens des années 50, 60 et 70 le projet de fonder un programme de recherche qui porte sur les aspects du langage non traités par la linguistique saussurienne de son époque. Ce programme se développe, comme cela a été soutenu par Lähteenmäki (2005), à partir du texte « Les genres du discours » (19521953) dans lequel Bakhtine établit une distinction entre la proposition - unité de la langue - et l'énoncé - unité de l'interaction ou de la communication verbale. Bakhtine envisage en effet une approche complémentaire de ces deux unités : "L'étude de l'énoncé, en sa qualité d'unité réelle de l'échange verbal, doit permettre aussi de mieux comprendre la nature des unités de langue (de la langue en tant que système) - les mots et les propositions. » (1984[1920-1974], p. 272)

Cette distinction est à la base de l'esquisse d'une épistémologie programmatique qui apparaît explicitement en 1963 dans la seconde édition de Problèmes de la poétique de Dostö̈evski, à laquelle Bakhtine a ajouté le chapitre conclusif «Le mot chez Dostoïevski ». ${ }^{4}$ Il nous semble que c'est l'aboutissement d'un parcours de recherche en

${ }^{2}$ La slaviste russe Mika Lähteenmäki, dans son article « De l'interprétation des idées linguistiques de Bakhtine: le problème des textes des années 50 et 60 », soutient la même idée : "Bakhtine ne souhaite pas remplacer la linguistique par la métalinguistique, mais tente de fournir un compte rendu des aspects du langage qui sont ignorés, ou éliminés par abstraction, dans les comptes rendus purement linguistiques du langage. » (p. 190)

${ }^{3}$ Sur la notion de science galiléene en linguistique, nous renvoyons le lecteur aux livres Introduction à une science du langage (1989) de Jean-Claude Milner et Introduction à la lecture de Saussure (1997) de Simon Bouquet.

${ }^{4}$ Même si cet ajout est connu depuis des années, cette information apparaît de façon plus explicite dans l'article de Lähteenmäki (2005). 
groupe qui avait commencé dans les années 20. L'épistémologie d'une métalinguistique se fonde sur trois aspects : la complémentarité par rapport à la linguistique de son époque, la délimitation d'un objet de recherche et la proposition d'un champ de phénomènes à étudier.

L'exposition du projet d'une métalinguistique se présente en opposition à la linguistique de la langue. Bakhtine commence par reconnaître la validité du projet de recherche de cette dernière : «la langue en tant qu'objet spécifique de la linguistique obtenu en abstrayant tout à fait légitimement et nécessairement le mot de certains aspects de la vie concrète ». (1970 [1929/1963], p. 211). En d'autres termes, le domaine premier de la linguistique est la langue déconnectée des énonciations singulières et particulières, c'est ce qu'entend Milner, par exemple, lorsqu'il affirme que la linguistique et la grammaire s'intéressent aux propriétés du langage qui restent intactes lorsqu'un énoncé est déconnecté des conditions singulières de son énonciation. Dans un deuxième temps, Bakhtine présente la linguistique et la métalinguistique comme l'étude du même phénomène, le mot, sous des angles différents. Malgré cette proximité, selon lui, Bakhtine nous avertit que les deux domaines ne doivent pas se confondre. Enfin, la métalinguistique s'intéresse aux phénomènes de dialogue qui, tout en appartenant au domaine de la langue, ne se restreignent pas à elle, puisqu'ils sont aussi de nature extra-linguistique.

Le projet de fonder la métalinguistique bakhtinienne se fonde sur la délimitation de son objet d'étude : «Les rapports de dialogue (y compris les rapports de dialogue entre le sujet parlant et sa propre parole) sont l'objet de la métalinguistique.» (Bakhtine, 1970 [1929/1963], p. 212) L'édition française (Seuil, 1970) de ce texte traduit l'expression «rapports de dialogue» par «rapports dialogiques $\aleph^{5}$. Cette variation pèse lourdement sur la réception contemporaine de l'œuvre du Cercle en France. Ainsi, Bres (2005) fait la distinction entre le dialogal («alternance de tours de parole» qui s'oppose au monologal), et le dialogique (compris comme «l'orientation de l'énoncé vers d'autres énoncés » et s'opposant au monologique). Nowakowska (2005) remarque que Bakhtine «ne disposait pas en russe de la distinction entre dialogal et dialogique ». En ce que nous concerne, nous ne ferons pas la différence entre dialogal et dialogique. Les textes du Cercle accordant, comme on verra ensuite à propos des sphères, une grande importance à l'interaction entre les genres premiers (majoritairement composés en forme de dialogue) et les genres seconds, nous soutiendrons que la proposition de l'interaction verbale comme réalité fondamentale de la langue est à la base du développement de la notion de dialogisme qui, à notre avis, contient à la fois le sens de dialogal et de dialogique.

Les rapports de dialogue ou dialogiques - ou, plus simplement, le dialogisme sont bien à la base de toutes les catégories de Bakhtine et de son cercle depuis les années $20^{6}$. Ils sont la condition du sens qui se produit à travers la compréhension

\footnotetext{
${ }^{5}$ Les traductions espagnole, américaine et brésilienne utilisent respectivement "las relaciones dialógicas", 'dialogic relationships" et "as relações dialógicas".

${ }^{6}$ Sur les origines de la théorie du dialogue en Russie, voir les articles de Alpanov (2004) et surtout d'Ivanova (2003). D'une part, Ivanova soutient que Voloshinov - à qui elle attribue l'autorité du livre Marxisme et philosophie du langage - a été influencé par le linguiste russe Lev Jakubinskij, auteur de l'article «Sur la parole dialogale », publié en 1923. Cet article pose les bases d'une théorie du dialogue
} 
responsive - autrement dit: il n'y a pas de sens hors de l'intersubjectivité et des rapports dialogiques : «Le sens ne s'actualise pas tout seul, il procède de deux sens qui se rencontrent et entrent en contact. Il n'y a pas de 'sens en soi '. Le sens n'existe que pour un autre sens, avec lequel il existe conjointement. » (1984 [1970-1971], p. 366). Dans cette perspective, on comprend que Bakhtine et son Cercle ne construisent pas une théorie du sujet ${ }^{7}$, mais une théorie des rapports entre interlocuteurs, base de la formation de l'identité personnelle. Grübel synthétise ainsi cette idée : «La théorie du dialogisme de Bakhtine part du principe que les positions idéologiques de ceux qui prennent part au discours sont autonomes et ne se prêtent à une médiation intersubjective que dans l'action communicationnelle elle-même. La position du moi se constitue comme position à travers l'autre, qui n'accède de son côté à l'achèvement qu'à travers le moi qui parle. » (Grubel, 2005, p. 107)

Les rapports dialogiques, en tant qu'objet de la métalinguistique, se manifestent entre les énoncés et à l'intérieur d'un énoncé. Bref, tous les aspects constitutifs de l'énoncé sont de nature dialogique et ses frontières se définissent par l'alternance de sujets parlants - énoncés-sources en-deçà, énoncés-réponses au-delà. D’une part, l'énoncé a un contact immédiat avec les énoncés d'autrui : un énoncé établit des liens dialogiques - de reflet, de désaccord, de filiation - avec les autres énoncés de la sphère de la communication discursive à laquelle il appartient. D'autre part, l'énoncé suscite l'attitude responsive des interlocuteurs qui se manifeste par des énoncés. Enfin, le style et les procédés compositionnels de l'énoncé sont conditionnés par la réponse présumée du destinataire $^{8}$ : son degré d'information de la situation, ses opinions, l'ampleur de ses connaissances spécialisées, etc.

La méta(ou trans)linguistique se dessine par la délimitation d'un champ de phénomènes, ressortissant au langage littéraire, défini comme objet d'étude. Ces phénomènes ont en commun le trait suivant : "Le mot y a une orientation double vers l'objet du récit en tant que mot ordinaire et vers un autre mot, vers le parler d'un autre. »" L'orientation vers le référent et vers le mot de l'autre donne à Bakhtine les

qui sera reprise par Voloshinov en accentuant l'aspect sociologique de l'interaction verbale. D'autre part, Ivanova montre le rapport qui existe entre la parole dialogale et le contexte académique soviétique des années 20, en soulignant que les études de linguistique historico-comparative ont donné lieu à «l'intérêt pour la langue vivante, pour l'utilisation de la langue et la créativité langagière a fait naitre des recherches de nouveaux objets pour la linguistique. Le développement des nouvelles sciences - de la poétique et de la pragmatique, la pénétration de la philosophie marxiste et de la sociologie a transformé le paradigme des sciences humaines » (p. 179).

${ }^{7}$ Sériot (2005) soutient cette idée à propos de la théorie de Voloshinov sur la situation sociale.

${ }^{8}$ Brandist (2004) fait l'hypothèse de l'influence de Karl Bühler (1879-1963) - psychologue et psychiatre allemand, membre de l'Ecole de Würzburg de Psychologie - sur la théorie de l'énoncé chez Voloshinov et sur le développement de la théorie du dialogisme chez Bakhtine: «Bühler amended Marty's account of 'triggering' to argue that what was anticipated were not always certain mental processes in the receiver, but often the actions he or she may perform. This bound the 'triggering' and 'steering' of anticipated response, that is, reciprocal regulation, into the utterance itself, something that was later creatively built upon by Bakhtin in his account of dialogic relations. » (p. 105)

${ }^{9}$ Bakhtine, M. M. (1970 [1929/1963]), Problèmes de la poétique de Dostö̈evski, Paris, L'Age d'homme, p. 215. La traduction américaine manifeste plus clairement que «l'objet du récit», c'est bien le 
moyens de classifier des types de discours : premièrement, le mot orienté exclusivement vers son référent; deuxièmement, le mot objectif ou le mot d'une personne représentée, autrement dit les diverses variantes du discours rapporté ; troisièmement, le mot à deux voix, dans ses occurrences diversement orientées - parodie, stylisation, polémique, réplique de dialogue.

Ce dernier type, qui est à la source de la notion de polyphonie chez Dostoïevski, est l'objet privilégié de Bakhtine. Autrement dit, on voit bien dans cette typologie le privilège accordé au mot à deux voix : elle incorpore le projet de la stylistique de son époque (le premier type) et les phénomènes étudiés par Voloshinov dans la troisième partie de Le Marxisme et la philosopbie du langage. Bref, le projet de Bakhtine de fonder une métalinguistique opère bien, nous semble-t-il, une synthèse quant aux objets d'étude développés depuis les années 20 par lui et d'autres membres du cercle.

\section{Les genres du discours}

La notion de genre ${ }^{10}$ gagne en visibilité à partir du texte de Bakhtine des années 50 , époque à laquelle Bakhtine entreprend le début d'une esquisse de programme d'une méta(ou trans)linguistique, dont le genre serait partie constituante. En poursuivant ce but, l'auteur établit les différences entre les unités de la langue et les unités de l'échange verbal. Cette distinction lui permet de placer la question des genres du côté de l'énoncé et d'établir ses aspects constitutifs :

L'utilisation de la langue s'effectue sous forme d'énoncés concrets, uniques (oraux ou écrits) qui émanent des représentants de tel ou tel domaine de l'activité humaine. L'énoncé reflète les conditions spécifiques et les finalités de chacun de ces domaines, non seulement par son contenu (thématique) et son style de langue, autrement dit par la sélection opérée dans les moyens de la langue - moyens lexicaux, phraséologiques et grammaticaux -, mais aussi et surtout par sa construction compositionnelle. Ces trois éléments (contenu thématique, style et construction compositionnelle) fusionnent indissolublement dans le tout que constitue l'énoncé, et chacun d'eux est marqué par la spécificité d'une sphère d'échange. Tout énoncé pris isolément est, bien entendu, individuel, mais chaque sphère d'utilisation de la langue élabore ses types relativement stables d'énoncés, et c'est ce que nous appelons les genres du discours. (Bakhtine, 1984 [1952-1953], p. 265)

L'expression « genres du discours », traduction de l'expression russe « rečevye žanry », pose quelques problèmes. Sériot (2003) pense que le terme « discours » est une modernisation de la terminologie et préfère les termes «mot» ou «parole» qui ont l'avantage de l'opacité. Du coup, Bouquet a préféré le titre «Les genres de la parole»

référent du discours : «Discourse in them has a twofold direction - it is directed both toward the referential object of speech, as in ordinary discourse, and toward another's discourse, toward someone else's speech. » (p. 185)

10 Brandist (2003) soutient que l'article «Sur la parole dialogale» du linguiste russe Lev Jakubinskij, publié en 1923, a été à la source de la formulation de Bakhtine sur les genres. 
pour le numéro 153 de la revue Langages qu’il a dirigé. Bronckart (2004) soutient la distinction entre deux niveaux méthodologiques : les « sortes de discours » dans le sens d'activités langagières et les genres de textes. Cette distinction lui permet de refuser la correspondance biunivoque entre sortes de discours/activités et sortes de textes. Pour notre part, nous conserverons l'expression genres du discours en nous appuyant, d'un côté, sur la décision terminologique des traducteurs espagnols, américains, français et brésiliens, et, de l'autre, sur l'idée que la notion de genres du discours est au croisement des types d'interactions verbales et des types stables d'énoncé, autrement dit que la distinction entre «types d'interaction verbale » et " genres » de Voloshinov/ Bakhtine (1986 [1929]), pp. 95-96) est plutôt une distinction de nature méthodologique qu'une distinction relevant de l'essence du phénomène.

L'ouverture du texte de Bakhtine, citée ci-dessus, synthétise les principaux traits de la notion de genre, en tant que type stable d'énoncé. Ces éléments sont tous de nature méta(ou trans)linguistique. En conséquence, son étude dépasse l'analyse purement linguistique et l'approche du genre doit prendre en charge les aspects dialogiques et extra-linguistiques du langage. De plus, la compréhension des aspects du genre - son existence dans les domaines/sphères, son contenu thématique, son style et sa construction compositionnelle - ne s'épuise pas dans le texte de Bakhtine des années 50, mais il faut les repérer dans les textes de Bakhtine, Medvedev et Voloshinov des années 20 et $30^{11}$.

\subsection{Sphère}

Le domaine ou la sphère est le premier aspect du genre souligné par Bakhtine. Ces termes sont accompagnés d'expressions variées au cours du texte «Les genres du discours » (1984 [1952-1953]). Ainsi on trouve «sphère » dans les syntagmes suivants : "sphère d'utilisation de la langue ", "sphère d'activité humaine », " sphère d'échange », «sphère culturelle », «sphère de l'échange verbal ", «sphère de l'échange culturel », "sphère de l'activité et de la communication humaine».

Malgré cette présence insistante du terme "sphère », on n'en trouve aucune théorisation éclairante dans ce texte ni dans les autres textes de Bakhtiniens. Le passage suivant nous donne cependant quelques pistes :

\footnotetext{
${ }^{11}$ L'autorité disputée des œuvres The formal method in literary scholarship et Le marxisme et la philosophie du langage est, de plus en plus, attribuée respectivement à Medvedev et à Voloshinov. En ce qui concerne le premier livre, Iurii Medvedev et Dar'ia Medvedeva (2004) afirment : «The work by Medvedev with the greatest resonance, undiminished to this day, is 'The Formal Method in Literary Scolarship : A Critical Introduction to Sociological Poetics', written in dialogue with Bakhtin. » (p. 43)

Par rapport au second livre, l'autorité de Voloshinov est renforcée par la traduction, dans le livre The Bakbtin Circle: in the master's absence (2004), de deux manuscrits de Voloshinov qui sont nettement à la source de Le marxisme. Il s'agit d'un résumé de la thèse soutenue par Voloshinov en 1929 et d'un rapport de recherche, tous les deux retrouvés dans L'Institut pour l'Histoire Comparée des Littératures et Langues Orientales et Occidentales à Saint Pétersbourg où Voloshinov a travaillé comme doctorant et chercheur salarié durant la deuxième moitié des années 20. Bien que ces livres aient été écrits par trois auteurs différents, les slavistes - comme Lähteenmäki (2005), Iurii Medvedev et Dar'ia Medvedeva (2004) - s'accordent à dire que les trois œuvres partagent la même conception du langage et de la production discursive.
} 
Le lien indissoluble, organique, du style et du genre apparait aussi avec une grande netteté lorsqu'il s'agit du problème d'un style de langue ou de fonction. En fait, le style de langue ou de fonction n'est rien d'autre que le style d'un genre propre à une sphère donnée de l'activité et de la communication humaine. Chaque sphère connait ses genres, appropriés à sa spécificité, auxquels correspondent des styles déterminés. Une fonction donnée (scientifique, technique, idéologique, officielle, quotidienne) et des conditions données, spécifiques pour chacune des sphères de l'échange verbal, engendrent un genre donné, autrement dit, un type d'énoncé, relativement stable du point de vue thématique, compositionnel et stylistique. (1984[1952-1953], p. 269).

On comprend mieux cet argument de Bakhtine en se rapportant au contexte historique de la production de ce texte. Selon Dolinin (2003), la stylistique fonctionnelle était, après la fin du marxisme, le paradigme dominant dans la linguistique russe des années cinquante. Son principe posait que la communauté des lieux de réalisation (littérature, presse, science, religion, etc.) produit une communauté typologique des propriétés linguistiques communes sous-jacentes qui demandent à être dévoilées. Selon Dolinin toujours, le texte de Bakhtine sur les genres du discours est une alternative à cette proposition en faisant «l'appel à l'approche inductive, à partir des genres concrets ». De cette lecture, il s'ensuit que Bakhtine prend en considération la classification des fonctionnalistes, pour la redévelopper. Tout d'abord, les fonctionnalistes et Bakhtine se rapprochent sur la question de l'identité des fonctions pouvant être attribuées aux sphères, à savoir les fonctions scientifique, technique, idéologique, officielle, etc., correspondant à des sphères de l'activité et de la communication humaine. Cependant, le projet de Bakhtine s'éloigne de celui des fonctionnalistes sur trois points: (1) il soutient que les sphères sont les lieux d'existence de plusieurs genres et, donc que les styles s'attachent plus aux genres qu'aux sphères, trop vastes et diversifiées pour n'recouvrir qu'un seul style; (2), la classification des genres doit se faire selon les sphères d'activité humaine, autrement dit que les sphères sont le principe de classification des genres ; (3), la différenciation des genres doit considérer la distinction entre genres premiers et seconds ${ }^{12}$.

La distinction et le rapport entre ces deux types est, d'après Bakhtine, de grande importance pour la compréhension de la théorie des genres. Il ne s'agit pas simplement de poser la question de l'hétérogénéité et du croisement des genres - en d'autres termes: qu'un genre est toujours dérivé d'autres genres ou qu'un genre absorbe et transforme d'autres genres. Sans négliger cet aspect, il nous semble que Bakhtine entend souligner, nous semble-t-il, trois éléments de sa conception du genre : la généralisation des résultats de ses recherches sur le roman, une approche théorique dialogique des genres, et l'enrichissement que l'analyse intégrée des sphères idéologiques et quotidiennes peut apporter à la théorie des genres.

\footnotetext{
12 La traduction espagnole rend plus visible l'importance de la distinction entre genres premiers et seconds dans le projet de Bakhtine : «Todo esto resulta de una falta de comprensión de la naturaleza genérica de los estilos. También influye la ausencia de una clasificación bien pensada de los géneros discursivos según las esferas de la praxis, así como de la distinctión, muy importante para la estilística, entre géneros primarios y secundarios. » (1952-1953/1982, p. 252).
} 
Dans ses études sur le roman, Bakhtine met l'accent sur la capacité qu’à celui-ci d'inclure les genres les plus diversifiés : «Le roman permet d'introduire dans son entité toutes espèces de genres, tant littéraires (nouvelles, poésies, poèmes, saynètes) qu'extralittéraires (études de mœurs, textes rhétoriques, scientifiques, religieux, etc.) » (1997 [1924-1970], p. 141). La caractéristique majeure du roman est justement cette hétérogénéité compositionnelle et stylistique : les différents genres incorporés par le roman se rapportent toujours à un style de langue, qui peut coïncider avec un dialecte social ${ }^{13}$, et à une structure compositionnelle. La littérature, et l'art en général, sont conçus comme des superidéologies, dans le sens qu'ils réfractent toutes les autres sphères idéologiques. Tihanov (2005) soutient que le cercle de Bakhtine a mis en évidence, en ce qui concerne cette conception de l'art comme une superidéologie, "une combinaison puissante de raisonnement néokantien et marxiste ». Bref, les conclusions de Bakhtine sur le roman en particulier reflètent sa théorie générale du genre: un genre se construit à travers d'autres genres.

La théorie du cercle est basée sur la conception du dialogue en tant que phénomène naturel du langage. Dans le texte sur les genres, le dialogisme interactionnel et interdiscursive est issu de l'observation des répliques du dialogue et conçu comme constitutif de tout énoncé. Ce sont les genres premiers auxquels appartiennent majoritairement les genres parlés/dialogués, qui expriment de la façon la plus nette le caractère responsif de tout énoncé. La langue écrite et les genres seconds absorbent les genres premiers, ce qui implique la présence plus ou moins marquée des tonalités dialogiques dans les genres seconds :

Dans leur grande majorité, les genres littéraires sont des genres seconds, complexes, qui sont composés de divers genres premiers transformés (répliques de dialogue, récits de mours, lettres, journaux intimes, documents, etc.). Ces genres seconds, qui ressortent d'un échange culturel complexe, simulent, en principe, les formes variées de l'échange verbal premier. (Bakhtine, 1984 [1920-1974], p. 307).

Ainsi, le rapport entre genres premiers et seconds permet-il d'expliciter le principe dialogique du langage, qui resterait dissimulé si l'étude des genres se concentrait exclusivement sur les genres seconds.

Enfin, l'importance accordée à la distinction entre genres premiers et genres seconds nous semble une conséquence de la théorisation du rapport entre l'idéologie du quotidien/psychologie sociale et les systèmes idéologiques. Dans le livre Le marxisme et la philosophie du langage, Voloshinov/Bakhtine cite la théorie de Plekhanov sur la psychologie du corps social en tant que maillon intermédiaire entre l'infra-structure et la super-structure : la psychologie du corps social existe dans les formes concrètes de l'interaction verbale qui se réalise dans les différents genres du discours.

\footnotetext{
13 Pour Lähteenmäki (2005), l'originalité de la théorie de Bakhtine sur le discours romanesque « réside dans son application de l'idée de stratification socio-idéologique du langage à son analyse du roman » (p. 188).
} 
Si la présence des genres a été effacée de la traduction française ${ }^{14}$, comme on peut remarquer ci-dessous, ceux-ci sont bien présents dans la traduction anglaise :

Social psychology exists primarily in a wide variety of forms of the "utterance", of little speech genres of internal and external kinds - things left completely unstudied to the present day. (...)

All these forms of speech interchange operate in extremely close connection with the conditions of the social situation in which they occur and exhibit an extraordinary sensitivity to all fluctuations in the social atmosphere. And it is here, in the inner workings of this verbally materialized social psychology, that the barely noticeable shifts and changes that will later find expression in fully fledged ideological products accumulate. (Volosinov, 1986[1929], p. 20).

La psychologie du corps social se manifeste essentiellement dans les aspects les plus divers de l' «énonciation » sous la forme des différents modes de discours, qu'ils soient intérieurs ou extérieurs. Ce domaine n'a été l'objet d'aucune étude jusqu’à présent. $[\ldots]$

Ces formes d'interaction verbale sont très étroitement liées aux conditions d'une situation sociale donnée et réagissent de façon très sensible à toutes les fluctuations de l'atmosphère sociale. C'est ainsi qu'au sein de cette psychologie du corps social matérialisée dans le mot s'accumulent des changements et des glissements à peine sensibles, qui, plus tard, trouvent leur expression dans les productions idéologiques achevées. (Bakhtine/Voloshinov, 1977[1929], p. 39).

L'importance du rapport entre genres premiers et genres seconds, soulignée par Bakhtine dans le texte des années 50 , tient aussi au fait que le projet du cercle est de relire la théorie marxiste de la superstructure, regardant la mobilité des idéologies constituées, conséquente à l'influence de l'idéologie du quotidien ${ }^{15}$. La nouveauté du livre de Voloshinov/Bakhtine tient à sa thématistion des contacts entre les différents systèmes idéologiques constitués à travers l'idéologie du quotidien et l'intérêt qu'il accorde aux genres quotidiens :

Les systèmes idéologiques constitués de la morale sociale, de la science, de l'art et de la religion se cristallisent à partir de l'idéologie du quotidien, exercent sur celle-ci une forte influence en retour, et donnent ainsi normalement le ton à cette idéologie. Mais

\footnotetext{
${ }^{14}$ Nous avons appris l'existence du terme « genre » dans l'oeuvre Le marxisme et la philosophie du langage à travers un entretien à Lausanne avec le slaviste Patrick Sériot et à travers la lecture de l'article de Souza (2003), "Gêneros discursivos em Marxismo e Filosofia da Linguagem” ("Genres discursifs dans Le marxisme et la philosophie du langage »). Cette information nous a amené à consulter les versions en espagnol et en anglais du texte de Voloshinov.

15 Pour une discussion approfondie des origines de l'idéologie du quotidien dans l'œuvre du cercle, lire l'article Tihanov, G. (2005), «L'Idéologie et le langage chez Vološinov : comment l'esprit de la Lebensphilosophie a engendré la sociologie marxiste» in Zbinden, K., Henking, I. W. (dir.), La quadrature du cercle Bakbtine : traductions, influences et remises en contexte, Lausanne, Centre de Traduction Littéraire, pp. 125-167.
} 
Sheila Vieira de Camargo Grillo

en même temps, ces produits idéologiques constitués conservent en permanence un lien organique vivant avec l'idéologie du quotidien, ils se nourrissent de sa sève, car, en dehors d'elle, ils sont morts, comme sont mortes, par exemple, l'œuvre littéraire achevée ou l'idée cognitive si celles-ci ne sont pas soumises à une évaluation critique vivante. (Bakhtine/Voloshinov, 1977 [1929], p. 131).

On comprend mieux, dès lors, la place cruciale réservée par Volochinov/ Bakhtine, dans les années 20, aux rapports entre genres premiers et seconds, rapportés à la notion de sphère. Tihanov met en évidence l'influence de Plekhanov sur cette idée du cercle : «Plekhanov remet déjà en question cette possibilité en avançant l'argument selon lequel l'infrastructure et les différentes idéologies sont liées non pas par une relation directe de cause à effet, mais plutôt par une relation de médiation avec la mentalité du peuple» (Tihanov, 2005, p. 129). Cette notion entraîne un éclatement de la vision marxiste orthodoxe des systèmes idéologiques, en déplacant l'accent sur leurs échanges et leur éternelle instabilité, plutôt que sur l'indépendance entre les sphères et leur rapport univoque avec l'infra-structure économique.

\subsection{Construction compositionnelle}

Des trois aspects du genre chez Bakhtine, la construction ou forme compositionnelle est considérée comme le plus caractéristique, ainsi que le souligne le terme «surtout» présent dans la définition de genre du texte des années 50 : «L'énoncé reflète les conditions spécifiques et les finalités de chacun de ces domaines, non seulement par son contenu (thématique) et son style de langue [...], mais aussi et surtout par sa construction compositionnelle.». Cet aspect du genre est caractérisé comme «type de structuration et de fini d'un tout» et comme «articulations compositionnelles ». Il est thématisé, d’une part, par la discussion établie dans les années 20 avec l'esthétique matérielle et, d'autre part, par son insertion dans le projet de la métalinguistique.

Dans le texte de 1924 «Le problème du contenu, du matériau et de la forme dans l'œuvre littéraire » (1997[1924-1970]), Bakhtine critique l'esthétique matérielle et la méthode formelle pour fonder une science empirique positive de l'œuvre d'art, en s'appuyant sur le principe de la primauté du matériau. Selon lui, ce principe peut produire des éclairages féconds sur la technique de l'œuvre d'art, mais il est insuffisant pour « explorer l'œuvre globale dans sa singularité et sa signification esthétiques ». Parmi les problèmes de l'esthétique matérielle, Bakhtine identifie la confusion entre les formes architectoniques et compositionnelles.

Les formes architectoniques sont entendues, relativement à l'objet esthétique, comme «formes d'une vision artistique et d'une procédure d'achèvement du monde » (Bakhtine, 1984 [1920-1974], p. 201). Dans cette perspective, l'activité artistique relève de valeurs cognitives et éthiques et qui donnent sens au tout de l'œuvre littéraire ${ }^{16}$.

16 "Pour une présentation de la notion de forme architectonique chez Bakhtine", voir Sobral, A. Ético e estético ("Éthique et esthétique") in BRAIT, Beth. (Org.) (2005) Bakbtin : conceitos-chave. ("Bakhtine : mots-clés"), São Paulo, Contexto, pp. 103-122, et l'article de Bénédicte Vauthier dans ce numéro. 
La forme compositionnelle est la réalisation d'une forme architectonique à travers l'organisation d'un matériau. Dans le cas de l'œuvre littéraire, le matériau est le langage verbal. Cette organisation se joue au niveau de la totalité et de l'articulation des parties, dirigée vers un but. Regardant la totalité, Bakhtine cite comme exemples de formes compositionnelles les genres roman, drame, poème, récit, nouvelle : «Il faut considérer que toute forme architectonique est réalisée au moyen de procédés compositionnels définis; d'autre part, aux principales formes compositionnelles (celles de genre, par exemple), correspondent, dans l'objet esthétique réalisé, des formes architectoniques essentielles. » (Bakhtine, 1997[1924-1970], p. 35). Il convient de remarquer que, dans ce texte, le genre équivaut peu ou prou à la forme compositionnelle. Regardant l'articu-lation des parties, Bakhtine mentionne le chapitre, la strophe, le vers, le paragraphe, les lignes, les mots. En bref, la forme compositionnelle traverse l'ensemble de l'organi-sation textuelle. Cette conception sera reprise, articulée à la théorie de l'énoncé, dans le texte des années 50.

En termes épistémologiques, la construction/forme compositionnelle révèle la nature « métalinguistique » du genre. Bien que la forme compositionnelle appartienne à l'énoncé, elle ne peut pas être saisie par les méthodes de la linguistique de la proposition. Tout d'abord, son volume extrêmement varié dépasse la proposition, vue comme unité de la langue, et pose des problèmes méthodologiques à la linguistique : «L'une des raisons qui fait que la linguistique ignore les formes d'énoncés tient à l'extrême hétérogénéité de leur structure compositionnelle et aux particularités de leur volume (la longueur du discours)» (Bakhtine, 1984[1920-1974], p. 288). Ce type de reproche adressé à la linguistique est aussi présent dans l'œuvre Le marxisme et la philosophie du langage, ce qui dénote la cohérence du projet du Cercle. L'insertion de la construction/forme composi-tionnelle dans le projet de la métalinguistique est encore plus claire, lorsque la desti-nation à un allocutaire est présentée comme étant à la base de la structuration de l'énoncé. A ce propos, on retrouve, une fois de plus, une harmonie entre le texte de Bakhtine des années 50 et le texte disputé des années 20 Le marxisme et la philosophie du langage :

Tandis que je parle je prends toujours en compte le fond aperceptif sur lequel ma parole sera reçue par le destinataire : le degré d'information que celui-ci possède sur la situation, ses connaissances spécialisées dans le domaine de l'échange culturel donné, ses opinions et ses convictions, ses préjugés (de mon point de vue), ses sympathies et ses antipathies, etc. - car c'est cela qui conditionnera sa compréhension responsive de mon énoncé. Ces facteurs détermineront le choix du genre de l'énoncé, le choix des procédés compositionnels... (Bakhtine, 1984[1920-1974], p. 304).

A la base de la division du discours en parties, dénommées paragraphes dans leur forme écrite, on trouve l'ajustement aux réactions prévues de l'auditeur ou du lecteur. Plus l'ajustement à l'auditeur et la prise en compte de ses réactions sont faibles, plus le discours sera d'un bloc et moins il y aura de paragraphes. (Bakhtine/Voloshinov, 1977 [1929], p. 158).

L'organisation du tout et la division de ses parties sont subordonnées, comme cela apparaît dans ces citations, par les réponses/réactions présumées de l'auditeur - 
ce qui témoigne tout autant des distinctions issues des critiques faites à la méthode formelle, avec sa distinction entre forme architectonique et forme compositionnelle, que des origines du projet de la métalinguistique, présenté de façon plus développé en 1963 : étudier les rapports dialogiques, exprimés dans tous les aspects de l'énoncé et de ses genres, y compris leur construction/forme compositionnelle.

\subsection{Contenu thématique}

Le contenu thématique rend compte de l'élément sémantique du genre, autrement dit du rapport entre l'énoncé et l'objet du sens, ou entre les représentations linguistiques et les référents du monde. Dans le texte «Les genres du discours », ce rapport est lié à la possibilité ou non d'exhaustivité de l'objet du sens. Dans les cas des genres les plus standardisés, la subjectivité créatrice de l'auteur a peu d'espace pour s'exprimer, et ces genres tendent à un épuisement total de l'objet du sens. Comme exemples de genres fortement standardisés, Bakhtine cite les genres militaires, les commandements et les ordres, ou les genres quotidiens, les questions factuelles. Pour ce qui est des genres moins standardisés, le côté créatif de l'auteur s'exprime davantage, ce qui entraîne un achèvement partiel de l'objet du sens. A titre d'exemple, Bakhtine cite les genres scientifiques. Dans tous les cas, le thème de l'énoncé est conçu comme une façon d'appréhender et d'achever l'objet du sens.

Dans la chronologie bibliographique du cercle, les racines de la notion de thème du genre apparaissent dans le texte "The formal method in literary scholarphip" de 1928. Bien que son autorité soit de plus en plus attribuée à Medvedev, nous partageons la vision des slavistes selon laquelle la pensée générale du livre est en accord avec celle de Bakhtine et de Voloshinov. Sur ce point, comme on l'a vérifié à propos de la construction compositionnelle, le dialogue avec les formalistes est à la base de la définition de la position du cercle. Ce texte propose deux types de définitions du genre. D’une part, il est défini à partir de sa propriété compositionnelle comme «the typical form of the whole work, the whole utterance» (p. 129), ce qui ressemble à la position de Bakhtine sur le genre en tant que forme compositionnelle, présent dans le texte «Le problème du contenu, du matériau et de la forme dans l'œuvre littéraire» (1924). D'autre part, il est compris comme «the aggregate of the means for seeing and conceptualizing reality » (p. 137). C'est cette dernière conception qui est essentiellement développée dans ce texte, de sorte qu'on peut y trouver des explications plus éclairantes de ce qu'est le contenu thématique du genre :

In the first place, the work is oriented toward the listener and perceiver, and toward the definite conditions of performance and perception. In the second place, the work is oriented in life, from within, one might say, by its thematic content. Every genre has its own orientation in life, with reference to its events, problems, etc.

(...)

But the intrinsic, thematic determinateness of genres is no less important.

Each genre is only able to control certain definite aspects of reality. Each genre possesses definite principles of selection, definite forms for seeing and conceptualizing reality, and a definite scope and depth of penetration. (Bakhtine/ Medvedev, 1991 [1928], p. 131). 
Dans cette œuvre, la constatation que les formalistes se sont concentrés uniquement sur la finalisation compositionnelle est confrontée à la dimension sémantique/ thématique de l'énoncé. Le thème n'est pas le résultat de la somme des significations des différents éléments ou des parties d'une œuvre: il transcende le caractère componentiel du langage, en tant qu'élément attaché au niveau de la totalité de l'énoncé. De plus, il est subordonné aux conditions de l'échange verbal, ce qui revient à dire que l'aspect sémantique est indissolublement lié à l'aspect pragmatique des partenaires de l'énoncé. Enfin, les caractères cognitif et éthique sont liés, parce que le sens n'existe pas hors de l'intersubjectivité et hors de l'évaluation sociale. De nouveau, le thème est de nature responsive et concorde donc avec le projet de la métalinguistique d'étudier les rapports dialogiques.

\subsection{Style}

Le style est une notion complexe, qui prend des tonalités variées dans les différents ouvrages de Bakhtine et de son Cercle ${ }^{17}$. Dans la perspective qui est celle du présent article, nous nous concentrerons sur le rôle du style dans la constitution du genre.

Bakhtine rattache le style au genre en s'opposant à la stylistique fonctionnelle russe des années 50 qui, comme on l'a vu, liait le style aux sphères idéologiques. Dans le texte «Les genres du discours» (1984 [1920-1974]), pp. 263-308), Bakhtine définit le style du genre comme «la sélection opérée dans les moyens de la langue - moyens lexicaux, phraséologiques et grammaticaux ». Cette sélection est contrainte, d'une part, par les aspects dialogiques de l'énoncé - l'influence du destinateur, le lien avec les énoncés précédents d'une sphère déterminée, le rapport avec l'objet du sens; et, d'autre part, par le degré de standardisation et de normativité du genre.

En ce qui concerne les aspects dialogiques, le texte des années 50 représente à la fois une reprise et un élargissement des propositions développées par Voloshinov dans les années 20 et 30 .

Dans le texte "La structure de l'énoncé », Voloshinov argumentait que le style est déterminé par la situation et l'orientation sociale de l'énoncé. Pour lui, la situation est composée par trois aspects de l'énoncé qu'il appelle sous-entendus ou parties extraverbales: d'abord, l'espace et le temps; ensuite, le sujet, l'objet ou le thème de l'énoncé ; enfin, la position ou l'évaluation des interlocuteurs vis-à-vis de l'événement. La situation joue un rôle déterminant dans la constitution de l'énoncé et dans la définition de son sens. En outre, l'orientation sociale est entendue comme un type de rapport social et hiérarchique entre le locuteur et l'allocutaire, autrement dit l'orientation sociale est constituée par le rôle social et la distance hiérarchique entre les partenaires de l'énoncé (mari/femme, patron/employé, collègues de travail, etc.). C'est cette l'orientation sociale qui est posée par Voloshinov comme le principe fondateur de l'intonation et du style de l'énoncé :

17 Pour une exposition de la complexité de la notion de style chez Bakhtine et son cercle, voir Brait, B., "Estilo" ("Style") in Brait, B. (2005), Bakbtin : conceitos-chave (Bakbtine : concepts-clés), São Paulo, Contexto, pp. $79-102$. 
L'intonation, qui exprime l'orientation sociale, contribue seulement à déterminer les critères stylistiques selon lesquels sont choisis les mots et les expressions, mais elle ne se limite pas à leur attribuer tel ou tel sens, elle indique également leur place dans l'ensemble de l'énoncé, et les y distribue. (Voloshinov, 1981 [1930], p. 314).

Pour démontrer cette idée, Voloshinov analyse un extrait des Ames mortes de Gogol, dans lequel le style de l'énoncé d'un personnage est déterminé et élaboré en fonction de sa position hiérarchique inférieure par rapport à son interlocuteur, à la situation sociale et au genre. La situation et l'auditoire se manifestent dans la construction d'un genre, lui-même fondateur de la constitution du style. En d'autres termes, le genre établit les principes et les contraintes de constitution du style : «Un genre de communication quotidienne de ce type - genre historiquement constitué et achevé - ne laisse en effet que fort peu de place aux libres variations. » (Voloshinov, 1981[1930], p. 314).

Bakhtine reprend et amplifie ces notions de Voloshinov. Selon lui, le destinataire présumé agit sur le style de l'énoncé en deux sens. D'abord, comme Voloshinov l'avait déjà démontré, le style du genre est déterminé par le rapport social et hiérarchique entre les interlocuteurs. À ceci, Bakhtine ajoute le fond aperceptif du destinateur ou la présomption de son niveau de connaissances et ses opinions valoratives :

Tandis que je parle je prends toujours en compte le fond aperceptif sur lequel ma parole sera reçue par le destinataire (...) Ces facteurs détermineront le choix du genre de l'énoncé, le choix des procédés compositionnels et, enfin, le choix des moyens linguistiques, c'est-à-dire le style de mon énoncé. (Bakhtine, 1984 [1920-1974], p. 304).

Nous voyons que la définition bakhtinienne du «style du genre » en tant que choix des moyens linguistiques est une reprise de la position de Voloshinov qui, d'ailleurs, est beaucoup plus précis et complet en ce qui concerne la démonstration des procédés stylistiques, puisque ce dernier caractérise le style non seulement par le choix des mots, mais aussi par leur distribution et leur organisation dans l'ensemble de l'énoncé.

Corollairement, le texte de Baktine des années 50 amplifie les facteurs de détermination du style du genre, en leur adjoignant aussi les rapports entre les énoncés précédents et les énoncés postérieurs d'une sphère déterminée. La spécificité de Bakhtine par rapport à Voloshinov est de souligner le double rapport de l'énoncé : en tant que réponse aux énoncés antérieurs et en tant qu'anticipation de la réponse active des destinataires. De cette façon, Bakhtine met l'accent sur la position responsive du locuteur, orientée à la fois vers les énoncés déjà prononcés, avec lesquels celui-ci dialogue, et vers les énoncés à venir des interlocuteurs présumés. Cette double orientation est toujours attachée à une sphère d'échange verbal et donc à un genre déterminé.

En ce qui concerne le degré de standardisation, le genre conditionne l'apparition de l'expressivité de l'auteur matérialisée dans son style personnel. La standardisation peut être comprise comme le degré de contrainte qu'un genre impose à son locuteur : ainsi les genres plus standardisés laissent-ils moins de place à l'expression du style individuel du locuteur, tandis que les genres moins standardisés sont plus aptes à exprimer la subjectivité du locuteur. 
Bakhtine amplifie encore cette notion en postulant que l'apprentissage de la langue passe par l'apprentissage des genres dans lesquels elle prend existence et circule, même si le locuteur n'a pas conscience de l'existence des genres : « Apprendre à parler c'est apprendre à structurer des énoncés [...]. Les genres du discours organisent notre parole de la même façon que l'organisent les formes grammaticales (syntaxiques).» (Bakhtine, 1984 [1920-1974], p.285). On peut discerner dans cette idée les résonances de la théorie bakhtinienne du rapport entre le genre roman et les langages sociaux. Dans le texte «Du discours romanesque », le genre est présenté comme une des forces de stratification du langage, un genre pouvant correspondre à une utilisation précise de la langue, liée à une vision de monde particulière : «Cette stratification est déterminée avant tout par les organismes spécifiques des genres. Tels ou tels traits du langage (lexicologiques sémantiques, syntaxiques ou autres) sont étroitement soudés aux intentions et au système général d'accentuation de tels ou tels genres. [...]. » (Bakhtine, 1997 [1924-1970], p. 110). Pour en donner un exemple, Bakhtine cite les genres journa-listiques, littéraires, oratoires. Bref, le genre peut être compris comme un niveau de contrainte mais aussi de possibilités qui fonde la nature non homogène du langage.

\section{Conclusions}

L'investigation de la notion de genre du discours dans l'œuvre de Bakhtine et de son Cercle nous a conduite à quelques prises des positions méthodologiques. D'abord, l'utilisation de plusieurs traductions s'est imposée en fonction de notre non-accès au russe et aux problèmes des traductions françaises, surtout en ce qui concerne l'effacement du terme «genre» du livre Le marxisme et la philosophie du langage. Ensuite, les contextes socio-historique et théorique de l'œuvre, que nous avons considérés à travers les textes des slavistes consultés, ont été essentiels pour la constatation des influences marquant l'œuvre de Bakhtine et de son cercle et, du coup, pour la compréhension de l'œuvre. Troisièmement, la diversité des auteurs liés à l'œuvre bakhtinienne et les différences de leurs positions théoriques qui en résulte ne nous ont pas empêchée de trouver une grande convergence de pensée dans le corpus concernée, comme nous l'avons mentionné à plusieurs reprises. Enfin, la notion de genre ayant été développée au fil des années de la production intellectuelle de ces auteurs, sa compréhension passe également par la considération de l'histoire du développement de cette notion.

Si le projet d'une métalinguistique est présent de façon implicite dans toute l'œuvre étudiée, c'est dans les années 50 et 60 qu'elle se systématise et s'explicite. Le projet de fonder une discipline pour analyser les rapports dialogiques posés à la base de la constitution du sens, de l'énoncé, de l'intersubjectivité, est fondamental pour la compréhension de la notion de genre du discours. Comme nous l'avons montré, tous ses aspects constitutifs - la sphère, le rapport entre genres premiers et seconds, son contenu thématique, son style et sa construction/forme compositionnelle - sont tributaires d'une approche dialogique de l'énoncé.

Pour conclure, dans le texte des années 50 , Bakhtine relève deux aspects des genres : la prescription et la standardisation. D'une part, le caractère normatif du genre 
rend possible l'apprentissage du langage, l'agencement de ressources de la langue et toute possibilité de prise de parole. D'autre part, le degré de standardisation des genres entraîne la présence de l'expressivité du locuteur et, peut-on ajouter, de son influence sur les changements du genre. À partir de ces idées et de la compréhension du genre comme déterminé par de faisceaux de critères (Rastier, 2001), les genres postulés par le Bakhtine des années 50 sont des normes qui régissent la production et l'interprétation des énoncés, aux niveaux textuel, sémantique et phraséologique, au sein des différentes sphères de la culture.

\section{RÉFÉRENCES BIBLIOGRAPHIQUES}

BAjTín, M. M. (1982 [1920-1974]), Estética de la creación verbal, Argentina, Siglo veintiuno.

Bakhtin, M. M. ; Medvedev, P. N. (1991 [1928]), The formal method in literary scholarship : a critical introduction to sociological poetics, Baltimore/London, Johns Hopkins Press.

Bakhtine, M. M./Voloshinov, V. N. (1977 [1929]), Le marxisme et la philosophie du langage, Paris, Minuit.

Bajtín, M.M. (2003 [1979]), Problemas de la poética de Dostoievski, 2ème ed., Madrid, Fondo de Cultura Económica.

Bakhtin, M. M. (1984 [1963]), Problems of Dostoievsky's Poetics, Minneapolis, University of Minnesota Press.

Bakhtine, M. M. (1970 [1929/1963]), La poétique de Dostö̈evski, Paris, Seuil.

Bakhtine, M. M. (1970 [1929/1963]), Problèmes de la poétique de Dostoïvski, Lausanne, L’Age d'homme.

Bakhtin, M. M. (1997 [1929/1963]), Problemas da poética de Dostoiévski, Rio de Janeiro, Forense Universitária.

Bakhtine, M. M. (1997 [1924-1970]), Esthétique et théorie du roman, Paris, Gallimard.

Bakhtine, M. M. (1984 [1920-1974]), Esthétique de la création verbale, Paris, Gallimard.

Voloshinov, V. N. (1981 [1926]), «Le discours dans la vie et le discours dans la poésie », in Todorov, T. Mikhaïl Bakbtine : le principe dialogique. Suivi de Écrits du Cercle de Bakbtine, Paris, Seuil. pp. 181-215.

Volosinov, V. N. (1986 [1929]), Marxism and the philosophy of language, Cambridge, Harvard University Press.

Voloshinov, V. N. (1981 [1930]), «La structure de l'énoncé», in Todorov, T, Mikbaïl Bakbtine : le principe dialogique. Suivi de Écrits du Cercle de Bakbtine, Paris, Seuil. pp. 287-316.

\section{Autres références}

AdAM, J.-M. ; Herman, T. (dir.) (2001), "Genres de la presse écrite et analyse de discours », Semen, $\mathrm{n}^{\circ} 13$. 
Alpanov, V. (2004), "The Bakhtin circle and the problems in linguistics" in SHEPERD, D. ; Tikhanov, G.; Brandist, C. (éd.) The Bakbtin Circle: In the Master's Absence, Manchester University Press, UK.

Bernard-Donalds, M. (1994), Mikhail Bakbtin between phenomenology and marxism, Cambridge, Harvard University Press.

BouQueT, S. (dir.) (1997), Introduction à la lecture de Saussure, Paris, Payot. (éd.) (2004), Langages, (Les genres de la parole) Paris, Larousse, $\mathrm{n}^{\circ} 153$.

BraIt, B. (Org.) (2005), Bakbtin : conceitos-chave, São Paulo, Contexto.

BRONCKART, J.-P. (2004), «Les genres de textes et leur contribution au développement psychologique », Langages, $\mathrm{n}^{\circ} 153$, pp. 98-108.

BRES, J. (2005), Savoir de quoi on parle: dialogue, dialogal, dialogique; dialogisme, popyphonie... in BRES, J. et al. (dir.) Dialogisme et polyphonie: approches linguistiques. Bruxelles, De Boeck. Duculot. (Actes de colloque).

Clark, K. ; Holquist, M. (1984), Mikhail Bakbtin, Cambridge/ Mass., Harvard University Press.

Brandist, C. ; Tihanov, G. (éd.) (2000), Materializing Bakbtin : the Bakbtin circle and social theory, Hampshire, Palgrave.

BRANDisT, C. (2003), «Bakhtine, La sociologie du langage et le roman » in SERIOT, P. (ed.), «Le discours sur la langue en URSS à l'époque stalinienne (épistémologie, philosophie, idéologie) ", Cabiers de l'ILSL, no 14, Lausanne, UNIL, pp. 59-83.

Depretto, C. (dir.) (1997), L’héritage de Bakhtine, Bordeaux, PUB.

Dolinin, K. (2003), «Le réalisme socialiste en linguistique» in SERIOT, Patrick (éd.), «Le discours sur la langue en URSS à l'époque stalinienne (épistemologie, philosophie, idéologie) ", Cahiers de l'ILSL, no 14, Lausanne, UNIL, pp. 85-100.

GruBEL, R. (2005), « La philosophie de la communication créative de Bakhtine et les problèmes de sa réception » in ZBINDEN, K., Henking, I. W. (dir.) La quadrature du cercle Bakhtine: traductions, influences et remises en contexte, Lausanne, Centre de Traduction Littéraire, pp. 63-124

IVANOVA, I. (2003), «Le dialogue dans la linguistique soviétique des années 1920-1930» in SERIOT, P. (éd.) «Le discours sur la langue en URSS à l'époque stalinienne (épistémologie, philosophie, idéologie)», Cahiers de l'ILSL, n 14, Lausanne, UNIL, pp. 157-182.

LÄHTEENMÄKI, M. (2005), «De l'interprétation des idées linguistiques de Bakhtine: le problème des textes des années 50 et 60 » in ZBinden, K., Henking, I. W. (dir.), La quadrature du cercle Bakbtine : traductions, influences et remises en contexte, Lausanne, Centre de Traduction Littéraire, pp. 169-202.

Medvedev, I. ; Medvedeva, D. (2004), “The scholarly legacy of Pavel Medvedev in the light of his dialogue with Bakhtin" in SHEPERD, D. ; TikHAnOv, G. ; BrAndist, C. (éd.) The Bakbtin Circle: In the Master's Absence, Manchester University Press, UK.

MiLner, J.-C. (1989), Introduction à une science du langage, Paris, Seuil. 
Sheila Vieira de Camargo Grillo

Nowakowska, A. (2005), Dialogisme, polyphonie: des textes de M. Bakbtine à la linguistique contemporaine in BRES, J. et al. (dir.) Dialogisme et polyphonie : approches linguistiques. Bruxelles, De Boeck. Duculot. (Actes de colloque).

Rastier, F. (2001), Arts et sciences du texte, Paris, PUF.

SERIOT, P. (éd.) (2003), "Le discours sur la langue en URSS à l'époque stalinienne (épistémologie, philosophie, idéologie) ", Cabiers de l'ILSL, nº 14, Lausanne, UNIL.

(2005) «Bakhtine en contexte: dialogue des voix et hybridation des langues (le problème des limites)» in ZBINDEN, K. ; HenKInG, I. W. (dir.), La quadrature du cercle Bakbtine: traductions, influences et remises en contexte, Lausanne, Centre de Traduction Littéraire, pp. 203-225.

SHEPERD, D. (2005), «La pensée de Bakhtine : dialogisme, décalage, discordance » in ZBINDEN, K. ; HENKING, I. W. (dir.) La quadrature du cercle Bakbtine: traductions, influences et remises en contexte, Lausanne, Centre de Traduction Littéraire, pp. 5-25.

Sheperd, D. ; Tikhanov, G. ; Brandist, C. (éd.) (2004), The Bakbtin Circle: In the Master's Absence, Manchester University Press, UK.

SouzA, Geraldo Tadeu de (2003), "Gêneros discursivos em Marxismo e Filosofia da Linguagem" in SouZA-E-Silva, C. ; Brait, B. (dir.), The Especialist, São Paulo, vol. 24, p. 185-202, especial.

Tinanov, G. (2005), «L'Idéologie et le langage chez Vološinov: comment l'esprit de la Lebensphilosophie a engendré la sociologie marxiste " in ZBINDEN, K. ; HeNKING, I.W. (dir.), La quadrature du cercle Bakbtine: traductions, influences et remises en contexte, Lausanne, Centre de Traduction Littéraire, pp. 125-167.

Todorov, T. (1981), Mikhaïl Bakbtine : le principe dialogique. Suivi de Écrits du Cercle de Bakbtine, Paris, Seuil.

Zbinden, K. ; Henking, I. W. (dir.) (2005), La quadrature du cercle Bakbtine : traductions, influences et remises en contexte, Lausanne, Centre de Traduction Littéraire. 